\title{
NFC Antennas for Modern Wireless Communication Applications
}

\author{
Abdalfettah Asharaa ${ }^{1,2}$, Omer Arabi ${ }^{1}$, Majid Salim Ali Alkhambashi ${ }^{2}$, Yousef Dama ${ }^{3}$, Ali \\ A. S. Alabdullah ${ }^{1,4}$, Embarak M Ibrahim Elfoghi ${ }^{5}$, Issa Elfergani ${ }^{1}$, Jamal Kosha ${ }^{1}$, Isah \\ Danjuma $^{1}$, Buhari Mohammed ${ }^{1}$, Y. I. Al-Yasir ${ }^{1}$, Naser Ojaroudi Parchin ${ }^{1}$, Raed Abd- \\ Alhameed $^{1}$ \\ \{A.S.M.Asharaa@bradford.ac.uk\}
}

University of Bradford, Bradford, West Yorkshire, Bradford, United Kingdom ${ }^{1}$

College of Electrical Engineering, Bani Walid University, Bani Walid, Libya ${ }^{2}$

Faculty of Engineering, An-Najah National University, Palestine3

College of Electronics Engineering, Ninevah University, $\mathrm{Iraq}^{4}$

College of Electronic Technology Bani Walid Libya ${ }^{5}$

\begin{abstract}
This work focuses on 13.56MHz NFC antennaas that will satisfy the operational requirement in horizontal orientations. The wavelength of $13.56 \mathrm{MHz}$ systems corresponds to about $22 \mathrm{~m}$. However, the antenna size is a small fraction of that in size and the operating distance is in the range of the reader antenna's diameter. Hence, the interaction between the reader and the ring happens in the near field of the interrogator. This leads to the fact, that the fundamental working principle can be described by a transformer, the capacitive or inductive coupling between the reader and the ring. In order to achieve a good coupling, the structure is made of different coils aligned together according to the current flow to avoid the disturbance of the induced magnetic field. In short, the coupling and the magnetic field distributions between the two components, the reader and the ring are presented.
\end{abstract}

Keywords: Near Field Communications (NFC), NFC Antennas, Wireless Communication, NFC Forum, Contactless Systems, Inductive Coupling, Mobile Telephone Systems, Close Range Communication.abstract.

\section{Introduction}

NEAR Field Communications (NFC) is an important technology that plays a key role in the realization of many wireless communication applications. It is a new wireless short-range communication technique for data transmission between intelligent devices such as mobile phones by integrating a small NFC reader into cellular phones [1] [2]. It is a short-range halfduplex communication that was jointly developed by Philips and Sony in late 2002 for contactless communications [3] [4]. NFC relies on the inductive coupling principle between transmitting and receiving devices and differs from far-field RF communication which is used for longer-range wireless applications [5] [6].

In other words, NFC is a short-range wireless communication technology that emerged only a few years ago. It has rapidly gained appreciation as a significant contributor to several techniques such as modern wireless applications, wireless sensor networks, cloud computing, etc [7]. NFC is an upcoming technology developed over RFID, in a way that consists of an interface and protocol that is based on RFID which makes the NFC device a part of the standard and compatible with existing RFID technology. It is a new technology that enables a contactless wireless communication link between devices close to each other less than 10 centimeters for sharing information at a maximum data rate of $424 \mathrm{kbps}$. This communication can be either active, passive or both active devices which do NFC works by utilizing magnetic coupling between devices. NFC is a new paradigm for the vast majority of cell phone users and is emerging as a near-term reality. It has a growing business potential technology. For instance, it allows people to use their cell phones to pay their travel tickets or pay for their purchases instead 
of using their bank cards. Also, there have been many applications developed by this new technology such as electronic keys, identification, receiving and sharing information or applying it as a setup service. NFC provides the possibility that users can share business cards, make transactions, access information from smart posters or provide credentials for access control systems with a simple touch. Therefore, it can be said that NFC provides easy connections, quick transactions, and simple data sharing. The main technical feature of NFC is that it complements many wireless technologies in a way that utilizes the key parameters and elements in the existing standards for contactless card technology. The complementing features of enables NFC are that it can be compatible with other existing contactless infrastructures and able the users to use one device with different systems. As mentioned, NFC is initiated over RFID and has more communication possibilities. The main characteristic that differentiates NFC from RFID is that the new technology prepares bidirectional data transmission between NFC equipped devices [8] [9].

Nowadays, the developing and growing mobility of equipments provided by modern wireless communications is becoming an essential feature in the emerging technological world. Before NFC technology been known, mobile phones already had several types of communication options with the external environment. When mobile phones were introduced, the primary need was to set up voice communication provided by Global System for Mobiles (GSM) which has other services such as SMS, MMS, and even internet access. Later Bluetooth technology was introduced that connects peripherals with computing devices, including mobile phones. In the present day, NFC is becoming a popular technology in mobile smartphones. This technology needs two NFC compatible devices placed very near to each other in order to communicate. It can transmit information up to a maximum rate of $424 \mathrm{Kbits}$ per second [10]. In the two devices are needed, the first device, which is called the initiator, is an active device and is responsible for starting the communication. The second device is called the target, which responds to the initiator's requests. The target device may be active or passive. The communication starts when the active device gets close to the target and generates a $13.56 \mathrm{MHz}$ magnetic field and powers the target device. The NFC technology works via magnetic field induction and operates on an unlicensed radio frequency band. Also, it includes an embedded energy source component whereas the target can be an RFID card, tag or an NFC device which gives the reply to the initiator's request [11] [12].

Furthermore, it is possible to use NFC for many purposes that allow people to do all sorts of things, such as payment for goods, have access to various forms of transportation, and gain access control to buildings. We find smart cards all over the place, which really can become a problem. Filling our pockets with twenty or so smart cards makes working with them a nightmare. NFC-enabled devices solve this problem by storing the information needed in a single device that we can use in place of the smart card. NFC makes our life a lot easier. As an example, a common use for NFC is to pay for a train or a bus. London uses the Oyster card to give people the capability to tap their cards to gain access to the required transportation services [13] [14]. Moreover, the NFC these days is more important, because this technology has the potential to significantly change how we interact with the people and things around us. The exciting news about NFC is that we have not even scratched the surface yet of how this technology is going to change our lives [8]. NFC designs are are very usefull to enable more differnt exchanges between different participants. It can still read passive RFID tags with an NFC reader, and we can write to their limited amount of memory. NFC also allows us to write data to certain types of RFID tags using a standard format, independent of tag type. It can also communicate with other NFC devices in a two-way, duplex, or exchange. NFC devices can exchange information about each other's capabilities, swap records and initiate longer-term communications through other means. However, the important question to be asked is that why does the phone stream its audio over the NFC connection? Two reasons: first, the NFC connection is intentionally short-range, generally $10 \mathrm{~cm}$ or less. That allows it to be low-power and to avoid interference with other radios built into devices using it. Second, it is relatively low-speed compared to Wi-Fi, Bluetooth, and other communications protocols. NFC is not designed to manage extended high-speed communications. It is for short messages, exchanging credentials and initiating relationships.

What is exciting about NFC is that it allows for some short and sophisticated introductions without the hassle of exchanging passwords, pairing and all the other more complicated steps that come with those other protocols. That means that when you and your friend want to 
exchange address information from your phone to his, you can just tap your phones together. When we want to pay with our Google Wallet, we can just tap as we would an NFC-enabled credit card [8] [15]. When we are using NFC, our device does not give the other device to which it is speaking access to its whole memory; it just gives it the basics needed for exchange. We control what it can send and what it cannot and to whom. To sum up, various devices such as rings, watches, bracelets, clothes and other devices have been developed during the past and recent years. However, every technology comes with a few implementation issues. Some set of technical circumstances inevitably causes problems. Fortunately, this research work tries to present ideas that will fix many of them, such as providing a strong magnetic field of NFC antennas in those devices. Many applications of NFC are the extensions to current solutions. Wireless /Contactless payment and ticketing solutions are commonly available across the world and are compatible with NFC enabled devices. Taking these applications to an appropriately equipped mobile device will be the next step in the adoption of NFC [16] [17]. New generations gadgets such as iPad, iPhone and iPod are equipped with NFC technology. Recently, Microsoft announced that all Windows8 Phone devices would make use of the NFC technology. Google's Smartphone app, Google Wallet, allows users to load entire credit card information and pay with the swipe of their phone. Visa and Samsung combined to create an NFC compatible Smartphone which will carry special content that aims to make purchases faster.

In the Near Field Communication (NFC) research, many critical aspects remain unsearched and unexplored or require more specific study, although the NFC technology is currently thought to be well researched. Therefore, in this research, it was believed to be worthwhile to design and investigate NFC antennas in more detail as well as to classify and analyze the sensitivities. It is believed that this could lead to an optimal NFC antenna design.

\section{Antenna Designs}

Near field communication antenna design is one of the main challenges in the modeling, analysis and design of modern wireless communication systems. The main challenge is to be able to design an effective and efficient compact antenna with high sensitivity, low loss and achieve a good coupling at $13.56 \mathrm{MHz}$ spectrum band. This part demonstrates an important step for the design of the NFC antenna. As mention before, the approach followed in this design is to create and find an idea that serves in and parallel orientations of the ring antennas in this design. This means that to provide magnetic field coupling for parallel orientations, the ring antenna consists of two coils is presented. The FPCB rectangular coil is designed for perpendicular coupling. The curved circular coil is for parallel coupling. Two coils are connected in parallel and share the matching network. Furthermore, it is a challenging step to choose the items and explain the procedures for any designs. First of all, the total coil width will be $0.20 \mathrm{~mm}$, FPCB material is initially chosen to be $0.25 \mathrm{~mm} \mathrm{FR} 4$, and the ring material can be used for first design as Ceramic that has the relative permittivity $(\varepsilon r=30)$ and loss tangent $(\tan \delta$ $=0.01$ ). And also the ring size for this design is US11 and different sizes can be designed by the same steps as well. Moreover, the radius is equal to $10 \mathrm{~mm}$, and the width is equal to $13.05 \mathrm{~mm}$. The layout geometry of the proposed antenna (Dual Coil NFC Ring Antenna) is indicated in Figure 1. This work focuses on $13.56 \mathrm{MHz}$ HF NFC antennas simulation. The corresponding wavelength at $13.56 \mathrm{MHz}$ will be analyzed and discussed. Also, the antenna size will be explained and discussed in the size, and the operating distance is in the range of the reader antenna's diameter. The simulation results of the S-parameters for the design in different orientations will be considered and analyzed. Also, for more clarity of the coupling, the distribution of the magnetic fields between the rings and the reader will be presented with simulated results and figures. To conclude, the coupling and the magnetic field distribution between the two components, the reader and the ring will be investigated in clear detail. In this work, the NFC reader antenna structure can be seen in Figure 2, which consists of a double loop antenna with a length of $53.5 \mathrm{~mm}$ and a width of $53.5 \mathrm{~mm}$. it is placed in differential mode. To conclude, the initial PCB reader will be made of FR4 material that has a thickness of $1 \mathrm{~mm}$. 


\section{Antenna Simulations}

In this scenario, the ring is placed in parallel alignment to the reader, as shown in Figure 7, and the full circuit diagram is already shown in Figure 3. It is well-known that energy depends on how the electromagnetic field lines generated by the reader flow through the NFC tag antenna. From the latter point as well as from the results obtained from the next sections, it is confirmed that the finest results are achieved when both antennas are parallel and face each other. The detailed Matching impedance for the ports is shown in Table 1 and Table.2, where, the reader port impedance values are shown in Table 1, and Table 2 shows as well the FPCB ring port impedance values.

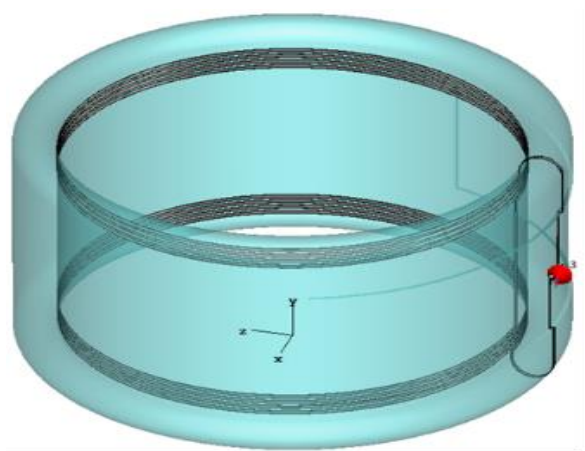

Curved circular coil

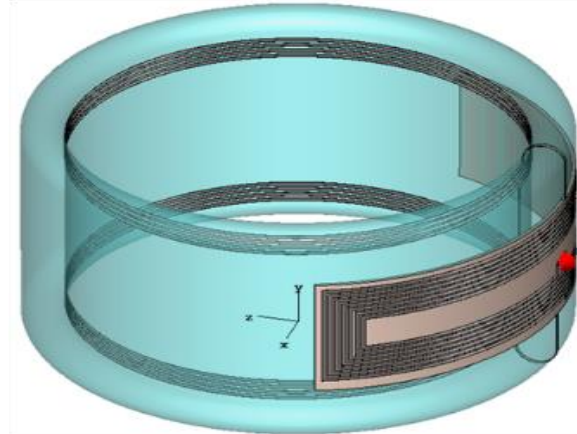

FPCB rectangular coil

Figure 1. Proposed NFC Ring Antennas.
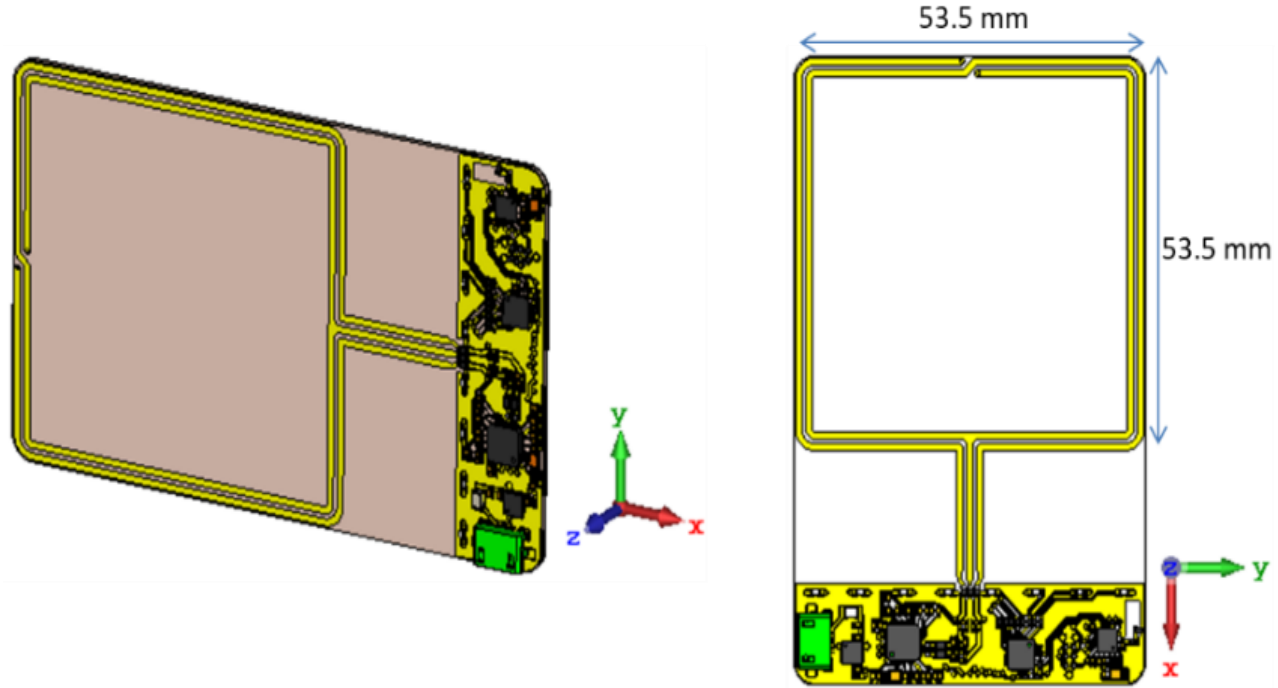

Figure 2. NFC Reader Antenna.

Table 1. Matching impedance.

\begin{tabular}{lcr}
\hline S. & Item & Value \\
\hline 1. & IC Impedance & $31 \mathrm{ohm}$ \\
2. & Cs1 & $281 \mathrm{pF}^{*}$ \\
3. & $\mathrm{Cp} 1$ & $217 \mathrm{pF}^{*}$ \\
\hline
\end{tabular}


Table 2. Dual coil ring port impedance.

\begin{tabular}{lcr}
\hline S. & Item & Value \\
\hline 1. & IC Impedance & $25 \mathrm{ohm}$ \\
2. & Cs2 & $120 \mathrm{pF}^{*}$ \\
3. & Cp2 & $150 \mathrm{pF}^{*}$ \\
& & \\
\hline
\end{tabular}

The dual-coil NFC ring specifications are shown in Table 3 and all dimensions of the structure of the coil NFC ring antennas are presented clearly in Figure 4, where are calculated in $(\mathrm{mm})$ millimeter.

Table 3. Substrate details used for the design.

\begin{tabular}{llll}
\hline S. & Item & Symbol & Type \\
\hline 1. & Ring size & S.ring & US11 \\
2. & Ring material & M.ring & $\begin{array}{l}\text { Ceramic }(\varepsilon r=30, \text { Loss tangent } \\
\delta=0.01)\end{array}$ \\
& & & \\
3. & Radius & R & $10.3 \mathrm{~mm}$ \\
4. & Width & W & $13.05 \mathrm{~mm}$ \\
5. & FPCB material & Mfcb & $0.025 \mathrm{~mm} \mathrm{FR4}$ \\
6. & Total coil width & Wcoil & $0.20 \mathrm{~mm}$ \\
& & & \\
\hline
\end{tabular}

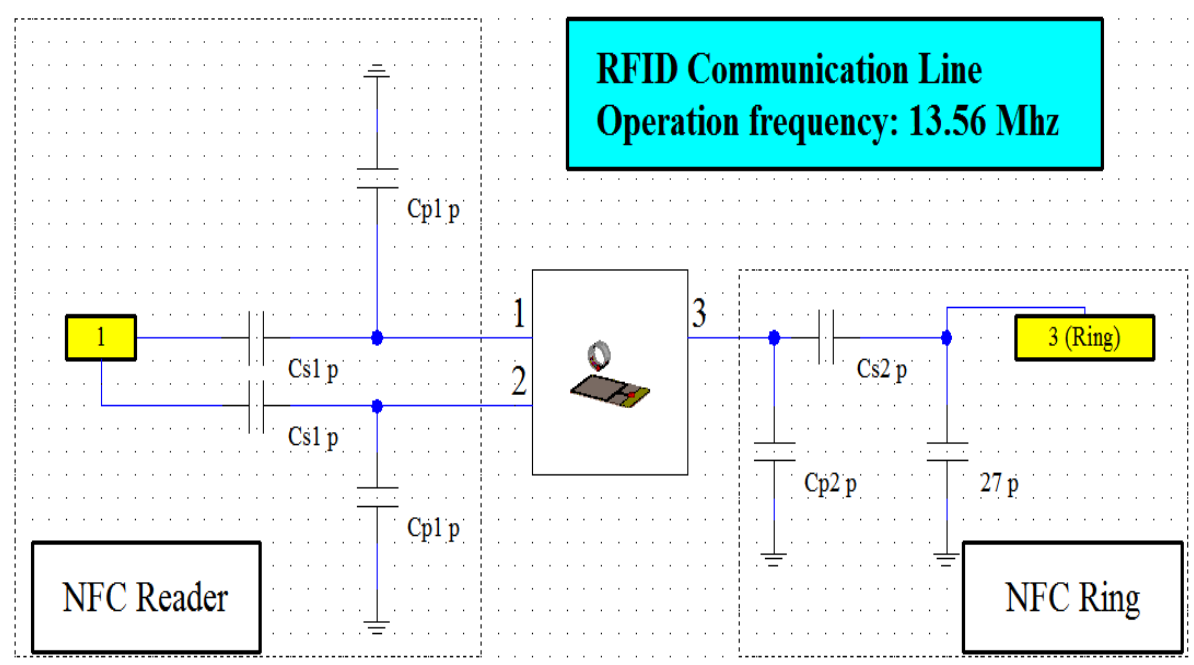

Figure 3: Circuit diagram. 


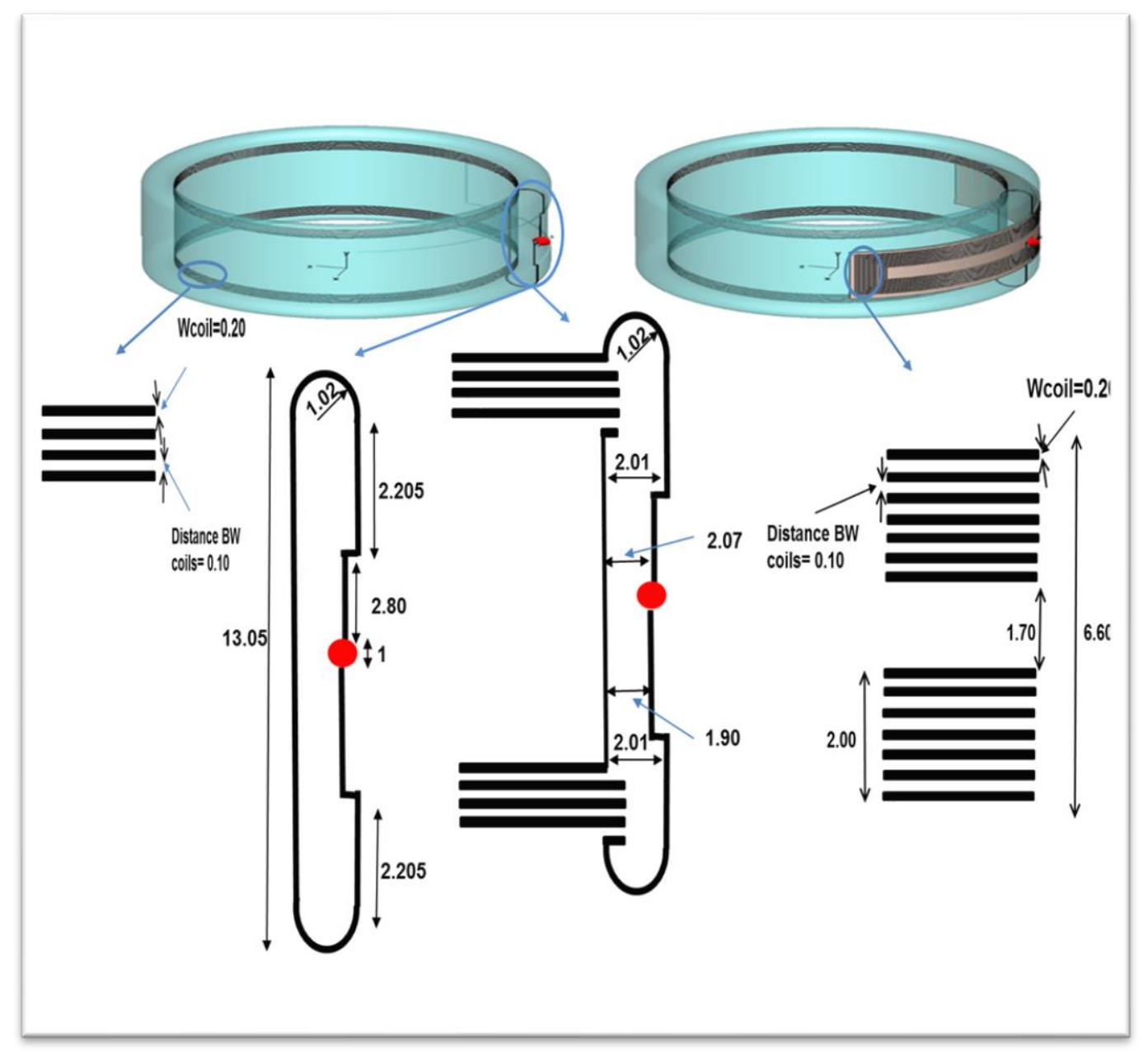

Figure 4: Dimensions of the structure of the-coil NFC ring antennas

It is clear that the previous figures have shown that the dual-coil NFC ring antennas structure with two ideas of building NFC antennas. This means that the design has a curved circular coil as can be seen in the left ring in Figure 4, where the full structure of the ring has an FPCB rectangular coil with the curved circular coil as shown in the right ring in that Figure above. Figure 5 shows the S-parameters when the ring IC chip impedance is matched and set with 20 $\mathrm{mm}$ spacing between the ring and the reader (orange solid line shown in Figure 5). The coupling reaches a very good results, As shown clearly down in Figure 6, which presented the correlation between the reader antenna and the ring antenna emissions. The spacing between the reader and the ring is set at $20 \mathrm{~mm}$, and the circuitry loss was not considered. In short, S-parameters and Smith chart of the dual coil ring and NFC reader in the parallel orientation illustrated in very good results, which expressed a high coupling between the ring and the reader. 

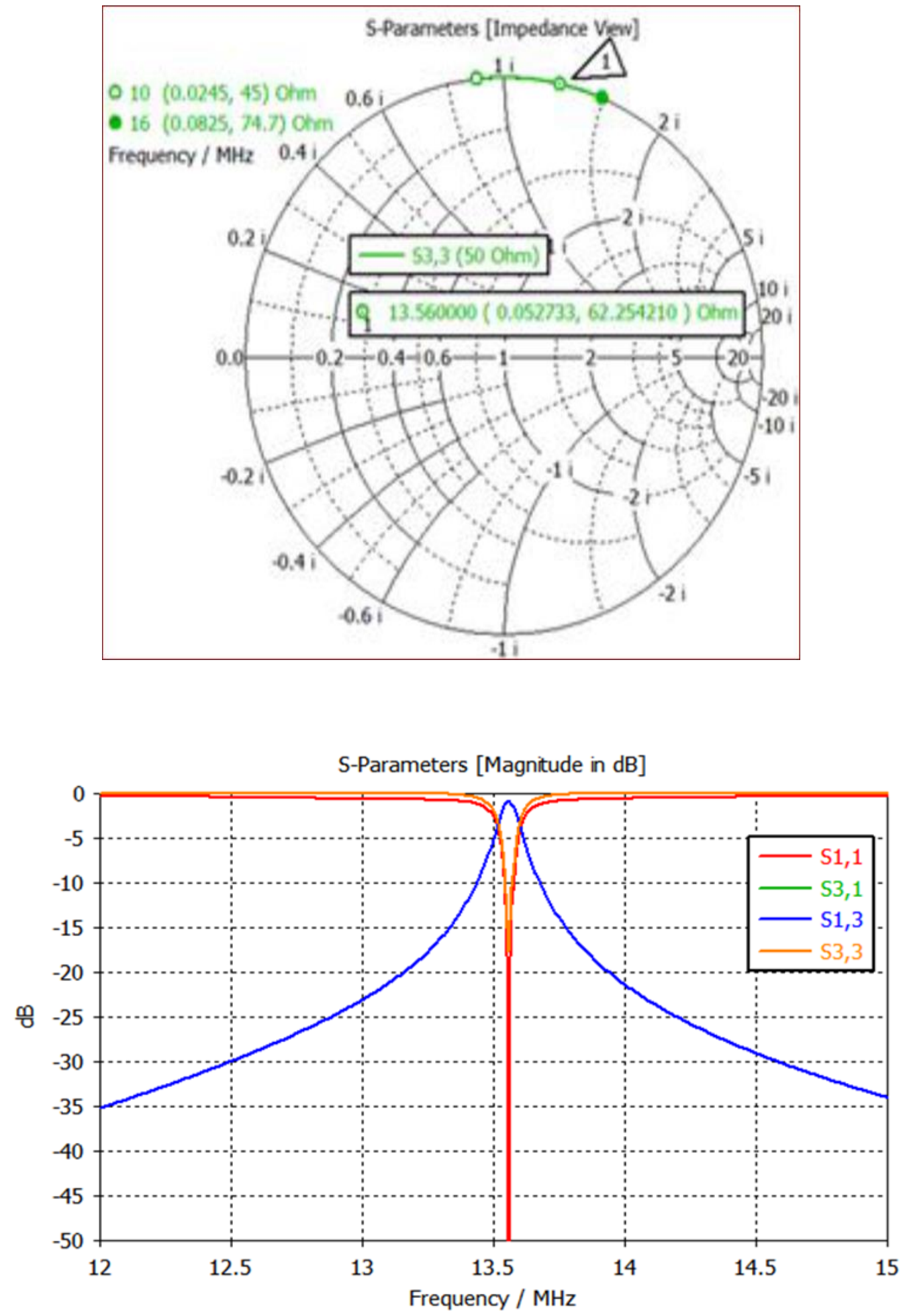

Figure 5: S-parameters and smith chart of the dual coil ring and NFC reader - parallel orientation. 
As shown clearly down in Figure 6, which presented the correlation between the reader antenna and the ring antenna emissions. The spacing between the reader and the ring is set at approximatley $20 \mathrm{~mm}$, and the circuitry loss was not considered.

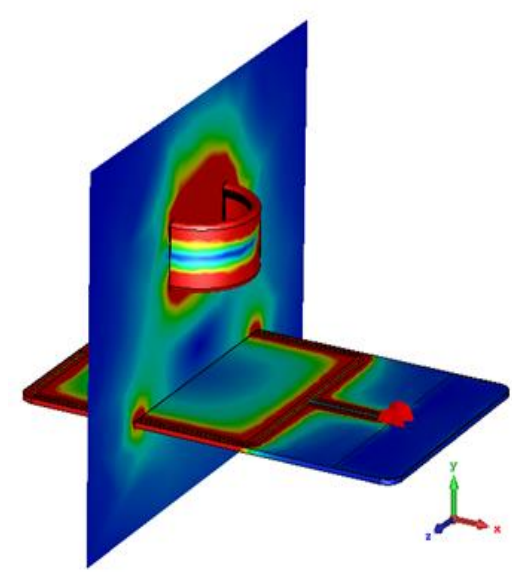

(a)

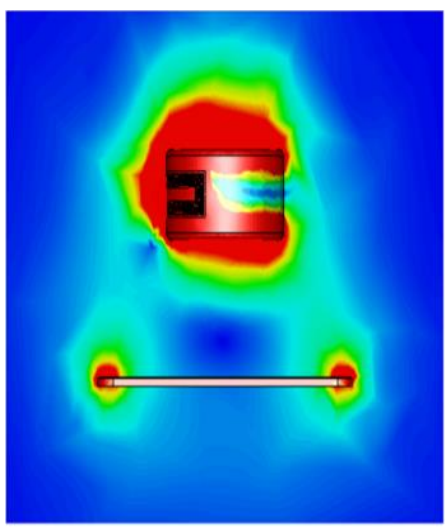

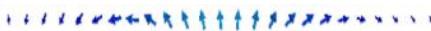

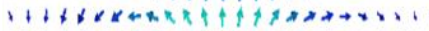

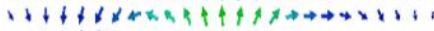

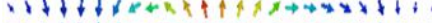

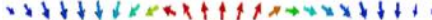

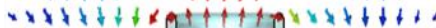

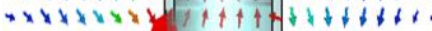

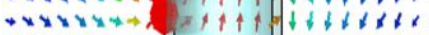

$\rightarrow \rightarrow x x x \rightarrow+\{11\}+10, x<x<x x$

$\rightarrow \rightarrow \rightarrow \rightarrow \rightarrow \rightarrow \rightarrow \rightarrow \infty x+1+4+\infty x<x<+\infty x+$

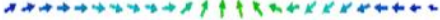

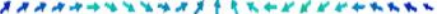

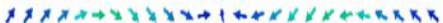

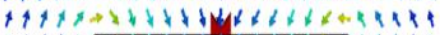

$t \uparrow t \uparrow t$ ard

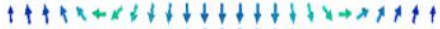

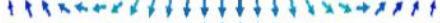

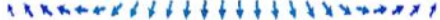

$x \times+40 x+6+b+t+b+8 x \times x \rightarrow \rightarrow+\infty$

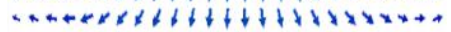

(b)

Figure 6: Magnetic fields strength distribution of the dual coil ring and NFC reader - parallel orientation: (a) full structure views, (b) magnetic field distribution.

\section{Analysis of The Design}

The proposed antenna has been analyzed using CST software [18]. The main goal and objective of the design are to measure and analyze the variation between the ring and the reader. Distance variation between the dual coil ring and the reader on the parallel orientation. On the parallel orientation, the distance (d) between ring and reader is shown in Figure 7 below, the distance (d) between the reader and the ring is varied as well at $20 \mathrm{~mm}, 10 \mathrm{~mm}$, and $5 \mathrm{~mm}$. The matching network and IC impedance remain the same as of Table 1 and Table 2. The obtained results in Figure 8 show the coupling level $(\mathrm{S} 1,3)$ at $13.56 \mathrm{MHz}$, which indicates a very good tolerance. 


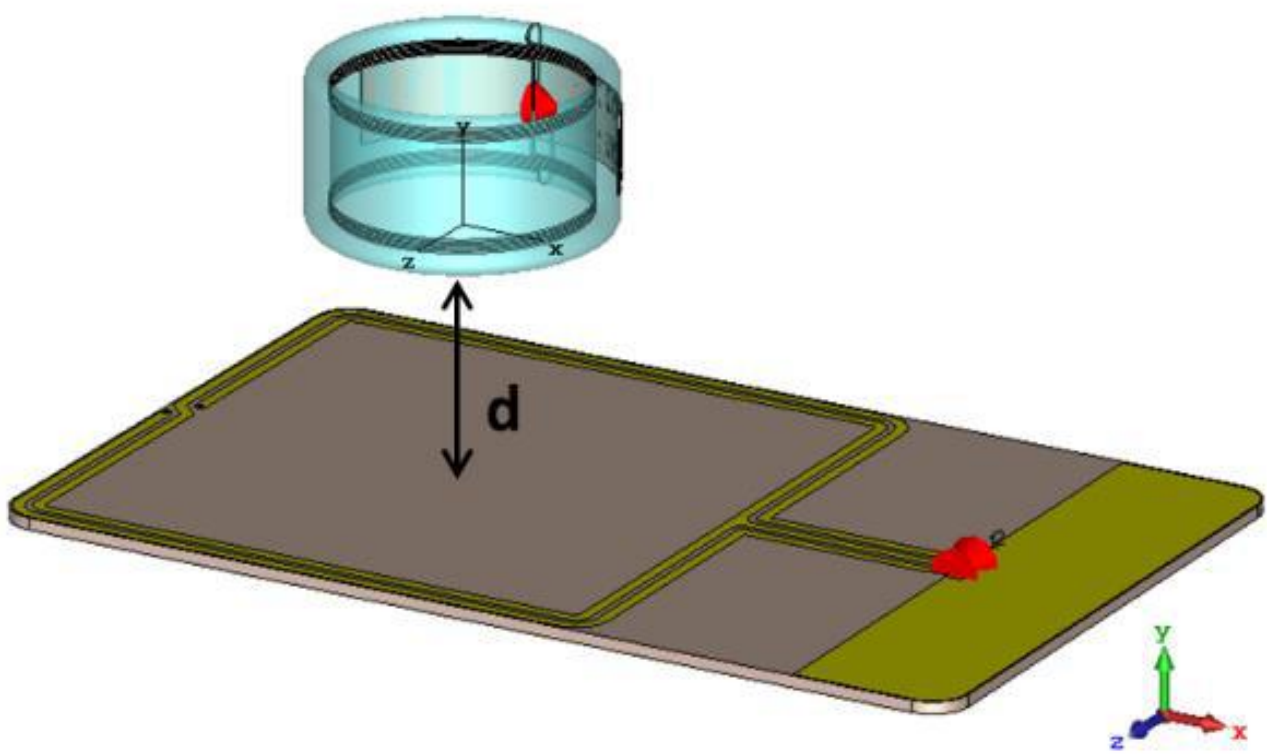

Figure 7: Dual coil ring and NFC reader - parallel orientation with distance d.

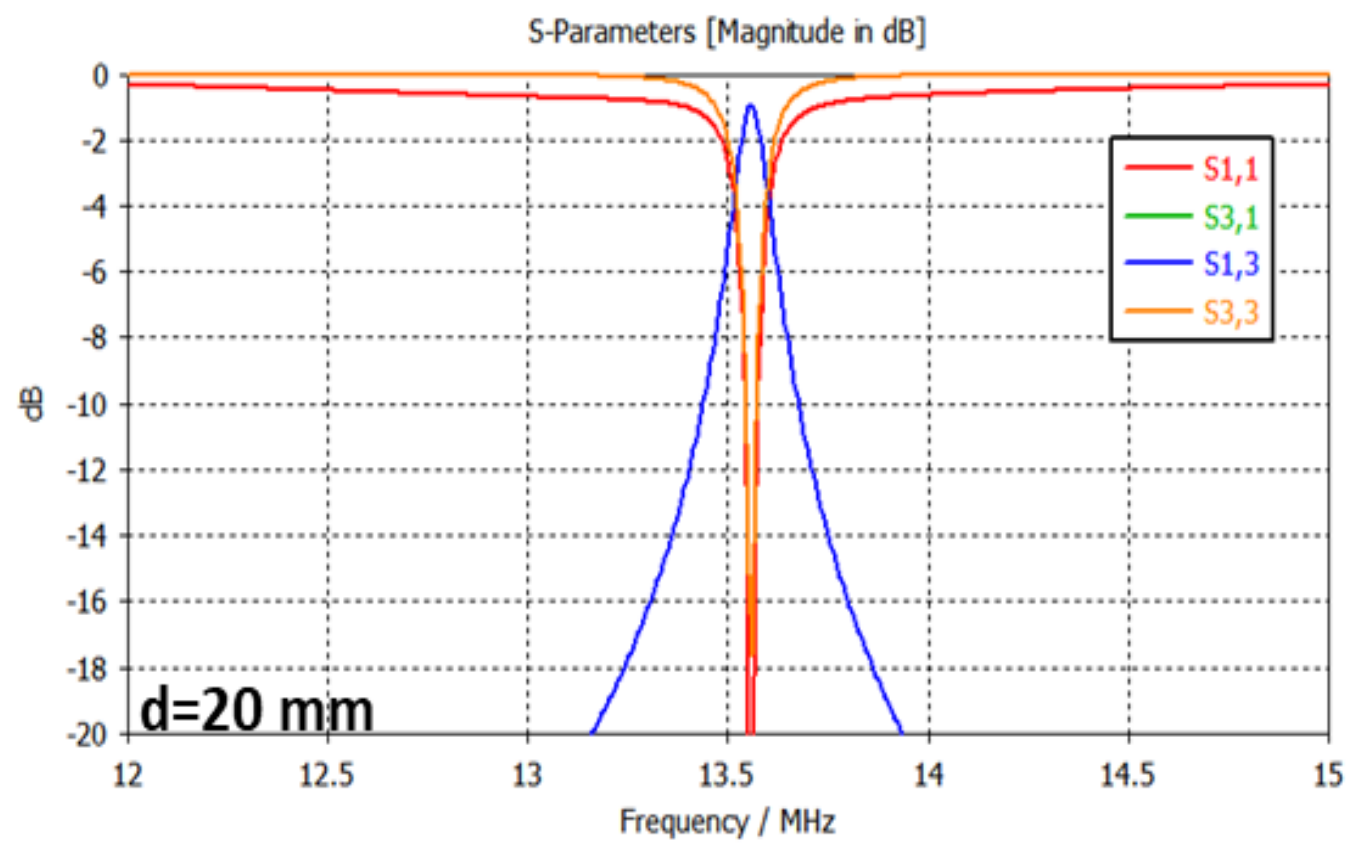

Figure 8: S-Parameters of the ring and NFC reader - parallel orientation at $\mathrm{d}=20 \mathrm{~mm}$. 


\section{5 . Conclusion}

This work demonstrated an innovative design and modeling of the NFC ring antenna that is capable of achieving high coupling characteristics in both perpendicular and parallel orientations with a loop transmitter reader. The systems are operating at a carrier frequency of 13.56 MHz, which serves as a base for Near Field Communication (NFC) applications. The NFC antennas designs were completed using CST software. These designs consist of several steps and procedures, which are planned step by step to obtain high performance. Strong magnetic field coupling achieved between the NFC reader and curved ring antennas in both configurations, parallel and perpendicular. To sum up, the final obtained results show good tolerance to distance variation.

\section{References}

[1] D. R. Walker and V. Moosavi, "Communication system providing near field communication (NFC) transaction features and related methods," ed: Google Patents, 2019.

[2] J. M. Park and K. T. Kim, "Near field communication terminal for performing secure payment and secure payment method using the same," ed: Google Patents, 2019.

[3] P. Verschueren and A. Kerselaers, "Antenna system for near-field magnetic induction wireless communications," ed: Google Patents, 2020.

[4] SONY. (2002). Philips and Sony announce a strategic collaboration to identify the next generation field radiocommunication. Available: https://www.sony.net/SonyInfo/News/Press_Archive/200209/02-0905E/

[5] B. S. Cook and S. Sankaran, "Contactless interface for mm-wave near field communication," ed: Google Patents, 2020.

[6] N. Hossein Motlagh, "Near Field Communication (NFC): A Technical Overview," 2012.

[7] B. JEPSON, D. COLEMAN, and T. IGOE, "Beginning NFC Near-Field Communication with Arduino, Android, and PhoneGap Jepson," ed: Tom O'Reillg Media, Inc, 2012.

[8] R. R. Sabella, NFC for Dummies: John Wiley \& Sons, 2016.

[9] M. Pulipati and S. Phani, "Comparison of Various Short Range Wireless Communication Technologies with NFC," Inter. J. Sci. Res, vol. 2, pp. 87-91, 2013.

[10] B. Ozdenizci, M. Aydin, V. Coskun, and K. Ok, "NFC research framework: a literature review and future research directions," in The 14th International Business Information Management Association (IBIMA) Conference. Istanbul, Turkey, 2010.

[11] V. Coskun, B. Ozdenizci, and K. Ok, "The survey on near field communication," Sensors, vol. 15, pp. 13348-13405, 2015.

[12] V. Coskun, B. Ozdenizci, and K. Ok, "A survey on near field communication (NFC) technology," Wireless personal communications, vol. 71, pp. 2259-2294, 2013.

[13] E. Strommer, J. Kaartinen, J. Parkka, A. Ylisaukko-oja, and I. Korhonen, "Application of near field communication for health monitoring in daily life," in 2006 International Conference of the IEEE Engineering in Medicine and Biology Society, 2006, pp. 3246-3249.

[14] R. P. Adhie, Y. Hutama, A. S. Ahmar, and M. Setiawan, "Implementation cryptography data encryption standard (DES) and triple data encryption standard (3DES) method in communication system based near field communication (NFC)," in Journal of Physics: Conference Series, 2018, p. 012009.

[15] V. Coskun, K. Ok, and B. Ozdenizci, Near field communication (NFC): From theory to practice: John Wiley \& Sons, 2011.

[16] V. K. Raina, U. Pandey, and M. Makkad, "Use of mobile transactions payment model in customer oriented payment system using NFC technology," International Journal of Mathematical and Computer Sciences, vol. 11, pp. 49-57, 2011.

[17] I. Jimena Medina, M. Gómez-Luque, J. Peña Amaro, I. Luque Ruiz, and M. Gómez-Nieto, "HistoNFC: An Innovative Tool for the Practical Teaching of Histology Using NFC Technology," Wireless Communications and Mobile Computing, vol. 2019, 2019.

[18] M. Studio, "Cst-computer simulation technology," Bad Nuheimer Str, vol. 19, p. 64289, 2008. 